\title{
Comparative Analysis of Indigenous Cloud Computing Providers in Nigeria
}

\author{
Festus Adeyinka Osuolale \\ Department of Computer \\ Science, School of Computing \\ The Federal University of \\ Technology, \\ Akure, Nigeria
}

\author{
Akindosu Joshua Akanle \\ Department of Computer \\ Science, School of Computing \\ The Federal University of \\ Technology, \\ Akure, Nigeria
}

\author{
Lolade Funmilayo Lawal \\ Department of Computer \\ Science, \\ School of Computing \\ The Federal University of \\ Technology, \\ Akure, Nigeria
}

\begin{abstract}
Cloud computing is not just a new technological phenomenal but the result of different existing technology evolving to make up the foundation of smart computing. The adoption of this technology has provided a great means of achieving efficient and sustainable online cloud services with significant reduction in maintenance cost. Cloud computing is becoming more relevant in Africa and in Nigeria as a whole due to its rapid evolution in the main stream, giving the indigenous IT professionals, government agencies and Large-Scale Organization (LSOs) new business potentials. This work presents analysis of major indigenous cloud computing solution providers with respect to their operations, deployment mode, availability, responsiveness, authenticity, speed, repudiation offer while enlightening users on the provider to select for their various service and creating more awareness about cloud computing in Nigeria.
\end{abstract}

\section{General Terms}

Comparative analysis

\section{Keywords}

cloud computing, indigenous cloud providers, comparative analysis, operations, awareness.

\section{INTRODUCTION}

Over the last few years, cloud computing paradigm has witnessed an enormous shift towards its adoption and it has become a trend in the information technology space as it promises significant cost reductions and new business potential to its users and providers. Infrastructure challenge, anxiety over safety and security of data and government policies are factors that stalled the cloud computing growth. As the demands on data centers keeps growing in terms of energy consumption, cooling system, infrastructure, space, qualified IT professionals and day-to-day running costs. Despite the lots of effort there has been a slowly dwindling reluctance to embrace this new technological trend in most of Nigeria's business circle. Cloud Computing can be described as the use of computing resources, hardware and software, which are delivered as a service over a network, usually the Internet. Internet has been a driving force towards the various technologies that have been developed. This can also be linked to being reluctant and resistant towards having technology assets hosted and managed by third parties [1]. One can argue that technological development doesn't necessarily go hand in hand with the demands of the end users, mainly everything done nowadays can be seen on shelves of big industries or in any other industry which may include the IT services is a pure innovation, which aims is to gain as many potential consumers as possible.

Cloud computing technology provides the best path towards achieving efficient and sustainable online data service to the target market. Due to the cost of maintenance there is need to outsource these functions to a third party to reduce capital and operational expenses. Major cloud providers such as Microsoft, IBM, Google, Cisco and others in the globe have put in place structures that drive the spread of cloud computing in Nigeria and other Africa countries by either providing the cloud services directly to organizations or in partnership with local IT firms for better integration and penetration. Cloud computing procreation could be traced to August 24, 2006 when Amazon publicly test ran its Elastic Computing Cloud (EC2) with the main intention of offering developers an infrastructure that could be hired to meet their IT needs [10]. Today, virtualization within networks has given rise to the evolution of cloud computing as it enables a dynamic data center to provide a pool of resources that can be exploited as needed by clients to meet their workloads and varying business demands. Cloud computing presents a shift of control from the traditional way of companies owing their respective data center/infrastructure to a resolve on resource sharing thereby cutting down the cost of running businesses. The service could be provided using an enterprise's data center, or that of a cloud provider.

Cloud computing, however is just rising and still gaining more popularity and widespread use during the past eight years, but the technological concept itself is not completely new. It is basically the result of a gradual development of utility or socalled "grid computing" (figure 1), which are based on the utilization of available assets and services through either ondemand or pay per use basis. The main difference here being that grid computing is concentrated mostly on the technical ground for fast, modular, and adjustable IT infrastructure, while cloud computing is aiming towards the business processes side of distribution and delivery of services on top of the existing infrastructure.

In this work, more emphasis are placed on the concept of cloud computing, with detailed discussions on the deployment and service models of cloud computing, impediments/challenges to its adoption especially in Nigeria, limitations, technologies, and characteristics 


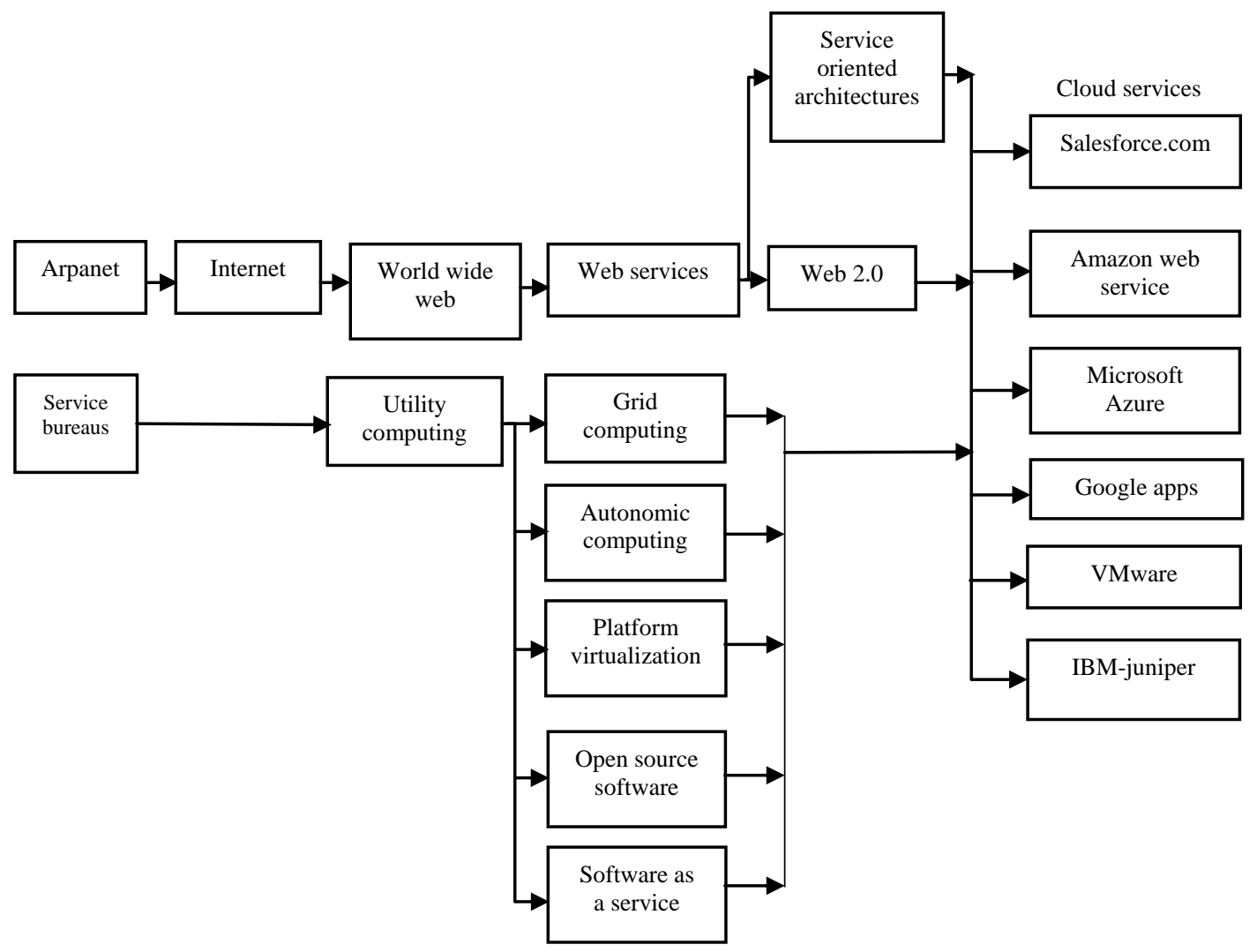

Fig 1: Origin of Cloud Computing [12]

\section{CLOUD COMPUTING}

Although many formal definitions have been proposed in both academia and industry, likewise different authors have given their opinion on the definition of cloud computing. The IEEE Standard Association (IEEE_SA) defines cloud computing in two working drafts "The P2301 (cloud profiles) draft highlight different ecosystem of cloud such as cloud vendors, service providers, and user. P2302 (Intercloud) drafts provides definition on topology, function, and governance for cloud-to-cloud interoperability and federation" [11], which is a group of information technology defines cloud computing as "A style of computing in which scalable and elastic ITenabled capabilities are delivered as service using internet technologies. [13] defines it as "computing on the Internet, as opposed to computing on a desktop", this definition succeeds since it recognize the dependency of cloud computing on the Internet [13]. Cisco, defines cloud computing as "IT resources and services that are abstracted from the underlying infrastructure and provided "ondemand" and "at scale" in a multitenant environment". Cisco definition points out three key attributes of cloud computing namely - on demand, at scale, and multitenant environment [4]. [1] defines cloud computing as "the dynamic provisioning of IT capabilities (hardware, software, or services) from third parties over a network". Just like Cisco it's states the "on demand", "at scale" and multitenant nature of cloud computing", [2], Also gave another definition of cloud computing which states that "cloud computing is the renting of infrastructure and software, as well as bandwidth, under defined service conditions. [2] These components should be adjusted daily to the needs of the customer and offered with the utmost availability and security. Included in cloud computing are end-2-end service level agreements (SLAs) and use- dependent service invoices". However, the definition is very comprehensive but rather too long.

Though Variants of cloud computing definitions exist and all definitions gave suitable clarification, still, the one with a broad acceptance among many IT and research agencies such as the Cloud Security Alliance (CSA), International Telecommunication Union (ITU) focus group on Cloud computing and the European Network and Information Security Agency (ENISA) among others is the definition from United States National Institute of Standard and Technology (NIST) which essentially defines cloud computing as "a model for enabling convenient, ondemand network access to a shared pool of configurable computing resources (e.g. networks, servers, storage, applications, and services) that can be rapidly provisioned and released with minimal management effort or service provider interaction". This definition includes cloud architectures, security and deployment and the ability for end users to utilize parts of bulk resources and that these resources can be acquired quickly and easily. The various definition of cloud computing differs across levels of organizations, yet it is based on the utilization of their facilities.

\section{CLOUD COMPUTING PROVIDERS IN NIGERIA (CCPN)}

\subsection{Layer3}

Layer 3 is a leading provider of information technology and telecommunication solutions partnering with global technology leaders such as juniper network, and so onAs a Juniper Elite Partner, Layer3 is positioned to deliver core IP infrastructure to service providers and mobile operators within Africa and it currently has one of the largest pool of Juniper certified experts in West Africa all with several 
years of experience in designing, deploying and supporting large and complicated network infrastructure. Layer3 currently possess the only Juniper Operate Specialist status in West Africa. Layer 3 partnership with other global leaders such as F5, Arbor Network, Blue Coat and Extreme Networks enables it to provide and support high performing, secure and robust networks [14].

\subsection{Mainone}

MainOne is a leading provider of innovative telecom services and network solutions for businesses in Nigeria and West Africa as a whole. MainOne's world class submarine cable system running down the coast of west Africa, state the art IP NGN network, growing regional and metro terrestrial fiber optic networks and data center facilities enable broadband services for businesses needing online connectivity solutions in West Africa. MDX-i Cloud Solutions provides a customized environment where your business can host their IT workload in an agile OPEX-based cloud model while ensuring your business remains compliant with unprecedented service levels that add good value to your business. Within West Africa's premier Tier III data center and only colocation facility with ISO 9001, 27001, PCI DSS and SAP Infrastructure Services certifications, MDX-i provides hybrid in-country Cloud services to a rich ecosystem of service providers [15].

\subsection{Computer Warehouse Group Plc}

Computer Warehouse group PLC birthed $26^{\text {th }}, 1992$ computer warehouse limited (CWL) catering mainly for to computer hardware projects. CWG is structured into various sector focused units in order to deliver improved products and services to its customers. Their services provided are includes: software services which include ERP solutions, banking solutions, data center infrastructure software; cloud services/ CWG 2.0 includes MFIs banking solutions, SMEs based ERP cloud solutions, co-location and hosting services; IT Infrastructure Hardware Services includes end user computing, servers and storage, data center hardware. CWG provides colocation services that allow organizations keep their data without upfront investment in hardware [6].

\subsection{Decasio Limited}

Decasio is one of Africa's leading digital transformation partner, housing some of the best world's cloud computing experts who transform organizations from analogue to digital, Application Modernization, Technical Support services, Digital Center Transformation and Cloud Migration so as to operate efficiently to achieve set business goals. Decasio partners with top technology OEMs i.e. Microsoft partner, Google and Amazon web services to provide unlimited access to the best cloud solutions for SMEs. Decasio cloud services is helping organizations around the world achieve flexibility, security, mobility and competitiveness while saving them a lot of money [7]

\subsection{Whogohost}

Whogohost is currently the largest and popular web hosting provider in Nigeria and the West African region. The company started as a web hosting firm but has grown to offer a lot of other products and services to help her customers grow and succeed online. Whogohost is an accredited NiRA (dotNG) registrar and also a Google G Suite accredited reseller. Cloud hosting is a type of virtual server running in a cloud computing environment. It is built, hosted and delivered via a cloud computing platform via the internet, and can be accessed remotely. This type of hosting is ideal for individuals and organizations that have a need for more resources or control than shared hosting can offer [2].

\subsection{Cloud Exchange West Africa (CEWA)} Cloud Exchange West Africa (CEWA) is currently building the first Tier IV data center in West Africa. Cloud Exchange SaaS Solutions decrease the time it takes to migrate applications to the cloud, speeding time to revenue and increasing return on investment. With a choice of cloud deployment models and a full range of services, rapid and safe transition can be done in the cloud and operate at max efficiency. It enables business agility with cloud services.

CEWA provide virtualized computing resources over the internet. Accompanying this is log access, security, load balancing and clustering, as well as storage resiliency, such as backup, replication and recovery. CEWA allow customers to develop, run, and manage applications without the complexity of building and maintaining the infrastructure. Customers' data are safe and secure with amazing security measures and vulnerability management. Cloud Exchange Cloud is highly secure, offering the users a fully managed environment along with compute, storage and network. CEWA's cloud infrastructure is highly robust and reliable [5].

\subsection{Digital Autonomy Information Technology Limited (DAIT)}

DAIT is an Information Technology firm which provides solutions to Internet related business problems. DAIT provide innovative IT solutions to clients from various industries, addressing their needs in today's highly competitive business environment. The solutions od DAIT are adaptive, scalable, secure and easy to use and support. DAIT strives to deliver cost-effective and adaptive technology solutions that enable her clients to meet their business objectives and goals, with returns on their investments. DAIT develop simple ICT solutions to highly complex problems thereby making it possible for easy solution deployment by their customers. With a team of certified professionals and renown international alliances, DAIT is poised to giving business owners the desired competitive edge. At DAIT, customer satisfaction is the mark of success [8].

\subsection{Galaxy Backbone Limited}

Galaxy Backbone is the information technology and shared services provider of the federal Government of Nigeria. A world class Uptime institute certified tier III data center located in Abuja with a disaster recovery site in Enugu has been built by this indigenous IT giant. Galaxy's service delivery model reflects the three service models defined by NIST which are infrastructure, software and platform as a service (IaaS, SaaS, and PaaS). Galaxy Backbone Limited is an Information and Communications Technology Services provider, wholly owned by the Federal Government of Nigeria. Galaxy Backbone continues to operate, improve and upgrade its common services platform to meet international standards. Infrastructure-asa-Service (IaaS) is an integral part of the service within Galaxy Backbone. Galaxy Backbone manages solution that allows agencies and customers to purchase their ICT 
infrastructure on demand. Purchasing these services on demand allows agencies realize cost savings and efficiencies while modernizing and expanding their IT capabilities without spending capital resources on infrastructure. Clients only pay for what they need, whenever required, without the burden of owning and maintaining their own infrastructure. Galaxy backbone limited provides the IT infrastructure required by Government agencies to be efficient and effective in delivering services to businesses and the citizens [9].

\subsection{Chert System Solutions Limited}

Chert System Solutions Limited is one of Nigeria's leading I.T Solution providers and a recognized cisco certified partner. They focus in providing IT products and services and as well as a growing portfolio of IT services which includes strategy assessment to installation of cost effective and scalable data center, provision of pre-sales support, device\& network monitoring to client, deployment of variety of network infrastructure services from switching and wireless to WAN applications and advanced network solution. Chert relationships with partners and its industry experience allow them to create scalable, cost effective Data Centre solutions for business in Nigeria. Chert works closely with her clients from strategy assessment to installation to ensure your data center meets your business objectives now and the future [13].

\section{SUMMARY OF THE CCPN FEATURES}

Features of few of the cloud computing providers whose operations are compared in this research are summarized in table 1 .

Table 1: Summary of features provided by CCPN

\begin{tabular}{|c|c|c|c|c|c|c|c|c|c|c|}
\hline & Layer3 & $\underset{\text { ne }}{\text { MainO }}$ & $\begin{array}{c}\text { CWG } \\
\text { ple }\end{array}$ & $\begin{array}{c}\text { Descasi } \\
\text { o Ltd }\end{array}$ & $\begin{array}{c}\text { WhoGo } \\
\text { Host }\end{array}$ & $\begin{array}{c}\text { Just } \\
\text { Host } \\
\text { Name }\end{array}$ & CEWA & DAIT & $\begin{array}{c}\text { Galax } \\
\text { y } \\
\text { Backb } \\
\text { one } \\
\text { Limite } \\
\text { d }\end{array}$ & CHERT \\
\hline $\begin{array}{c}\text { Cloud } \\
\text { Deployme } \\
\text { nt models }\end{array}$ & $\begin{array}{l}\text { Public } \\
\text { and } \\
\text { private } \\
\text { cloud }\end{array}$ & $\begin{array}{l}\text { Private, } \\
\text { Public, }\end{array}$ & $\begin{array}{c}\text { Private } \\
\text { and } \\
\text { public }\end{array}$ & $\begin{array}{l}\text { Private, } \\
\text { Public. }\end{array}$ & $\begin{array}{l}\text { Private } \\
\text { cloud, } \\
\text { Public } \\
\text { cloud }\end{array}$ & $\begin{array}{c}\text { Private, } \\
\text { Public }\end{array}$ & $\begin{array}{l}\text { Private } \\
\text { Cloud, } \\
\text { Public } \\
\text { Cloud }\end{array}$ & $\begin{array}{l}\text { Public } \\
\text { cloud }\end{array}$ & $\begin{array}{l}\text { Private } \\
\text { Public. }\end{array}$ & $\begin{array}{c}\text { Private, } \\
\text { Public }\end{array}$ \\
\hline $\begin{array}{c}\text { Service } \\
\text { model }\end{array}$ & $\begin{array}{c}\text { IaaS and } \\
\text { SaaS }\end{array}$ & IaaS & $\begin{array}{l}\text { Iaas, } \\
\text { SaaS }\end{array}$ & $\begin{array}{c}\text { SaaS, } \\
\text { paas, } \\
\text { IaaS, } \\
\text { xaas }\end{array}$ & IaaS & $\begin{array}{l}\text { Saas, } \\
\text { Iaas }\end{array}$ & $\begin{array}{l}\text { SaaS, } \\
\text { IaaS, } \\
\text { TaaS, } \\
\text { PaaS }\end{array}$ & $\begin{array}{c}\text { PaaS, } \\
\text { IaaS }\end{array}$ & All & $\begin{array}{l}\text { SaaS, } \\
\text { PaaS, } \\
\text { IaaS, } \\
\text { AaaS }\end{array}$ \\
\hline $\begin{array}{c}\text { Pricing } \\
\text { model }\end{array}$ & $\begin{array}{c}\text { Subscrip } \\
\text { tion }\end{array}$ & $\begin{array}{l}\text { Subscrip } \\
\text { tion }\end{array}$ & $\begin{array}{l}\text { subscrip } \\
\text { tion }\end{array}$ & $\begin{array}{l}\text { Pay-as- } \\
\text { you-go, } \\
\text { subscrip } \\
\text { tion }\end{array}$ & $\begin{array}{c}\text { Subscrip } \\
\text { tion }\end{array}$ & $\begin{array}{l}\text { Pay-as- } \\
\text { you-go } \\
\text { yearly } \\
\text { subscrip } \\
\text { tion }\end{array}$ & $\begin{array}{l}\text { Pay-as- } \\
\text { you-go, } \\
\text { subscrip } \\
\text { tion }\end{array}$ & $\begin{array}{l}\text { Subscrip } \\
\text { tion }\end{array}$ & $\begin{array}{c}\text { Per } \\
\text { annum }\end{array}$ & $\begin{array}{l}\text { Subscrip } \\
\text { tion }\end{array}$ \\
\hline $\begin{array}{c}\text { Virtualiza } \\
\text { tion }\end{array}$ & vSphere & Hyper-V & vSphere & $\begin{array}{c}\text { Microso } \\
\mathrm{ft} \text { VM. }\end{array}$ & $\begin{array}{c}\text { Xen } \\
\text { Hypervis } \\
\text { or }\end{array}$ & $\begin{array}{l}\text { Vsphere, } \\
\text { Mware } \\
\text { Hypervi } \\
\text { sor, Xen }\end{array}$ & $\begin{array}{c}\text { Xen } \\
\text { hypervis } \\
\text { or, } \\
\text { Solaris } \\
\text { containe } \\
\text { r } \\
\text { OpenSta } \\
\text { ck- } \\
\text { based }\end{array}$ & Xen & $\begin{array}{l}\text { VSphe } \\
\text { re }\end{array}$ & $\begin{array}{c}\text { Microso } \\
\text { ft CLR, } \\
\text { Open } \\
\text { VZ, and } \\
\text { Xen }\end{array}$ \\
\hline $\begin{array}{c}\text { Tools } \\
\underset{\text { /Framewo }}{\text { rk }}\end{array}$ & $\begin{array}{c}\text { Python, } \\
\text { PowerS } \\
\text { hell }\end{array}$ & SQL & Java & N/A & $\begin{array}{c}\text { Base on } \\
\text { the OS }\end{array}$ & $\begin{array}{c}\text { PHP, } \\
\text { Python, } \\
\text { Ruby, } \\
\text { Perl, } \\
\text { SQL }\end{array}$ & N/A & $\begin{array}{l}\text { Java, } \\
\text { PHP, } \\
\text { SQL }\end{array}$ & $\begin{array}{l}\text { Java, } \\
\text { SQL }\end{array}$ & $\begin{array}{c}\text { Python, } \\
\text { Ruby on } \\
\text { Rail, } \\
\text { Java }\end{array}$ \\
\hline $\begin{array}{c}\text { Availabilit } \\
\mathbf{y}\end{array}$ & $80 \%$ & 95 & 98 & N/A & 90 & 99 & 60 & 90 & 100 & 95 \\
\hline $\begin{array}{c}\text { Serviceabi } \\
\text { lity }\end{array}$ & $25 \%$ & 95 & 70 & N/A & 75 & 99 & 20 & 50 & 25 & 70 \\
\hline $\begin{array}{c}\text { Access } \\
\text { Interface }\end{array}$ & $\begin{array}{c}\text { Web } \\
\text { based, } \\
\text { console, } \\
\text { API }\end{array}$ & $\begin{array}{c}\text { Web- } \\
\text { based, } \\
\text { Console }\end{array}$ & $\begin{array}{l}\text { web- } \\
\text { based }\end{array}$ & $\begin{array}{l}\text { Web } \\
\text { based }\end{array}$ & $\begin{array}{c}\text { Web- } \\
\text { based } \\
\text { control } \\
\text { panel }\end{array}$ & $\begin{array}{c}\text { Web- } \\
\text { Based, } \\
\text { cPanel, } \\
\text { API }\end{array}$ & $\begin{array}{c}\text { Web- } \\
\text { Based, } \\
\text { API }\end{array}$ & $\begin{array}{c}\text { Web- } \\
\text { based } \\
\text { Control } \\
\text { Panel }\end{array}$ & $\begin{array}{l}\text { Web- } \\
\text { based, } \\
\text { Contro } \\
\text { l panel }\end{array}$ & $\begin{array}{c}\text { API and } \\
\text { Web- } \\
\text { based }\end{array}$ \\
\hline
\end{tabular}




\begin{tabular}{|c|c|c|c|c|c|c|c|c|c|c|}
\hline $\begin{array}{c}\text { Active } \\
\text { Service } \\
\text { Level } \\
\text { Agreemen } \\
\text { t (SLA). }\end{array}$ & Yes & Yes & yes & Yes. & Yes & Yes & Yes & N/A & Yes. & Yes \\
\hline $\begin{array}{c}\text { Penalties } \\
\text { associated } \\
\text { with SLA } \\
\text { noncompli } \\
\text { ance }\end{array}$ & $\begin{array}{l}\text { It is } \\
\text { stated in } \\
\text { the } \\
\text { terms of } \\
\text { service }\end{array}$ & $\begin{array}{l}\text { Service } \\
\text { credits }\end{array}$ & $\begin{array}{l}\text { suspensi } \\
\text { on }\end{array}$ & $\begin{array}{c}\text { Service } \\
\text { credit }\end{array}$ & $\begin{array}{c}\text { Suspensi } \\
\text { on for } \\
\text { defaulter } \\
\text { s }\end{array}$ & $\begin{array}{c}\text { Two } \\
\text { times } \\
\text { Notificat } \\
\text { ion, } \\
\text { Followe } \\
\text { d by } \\
\text { suspensi } \\
\text { on. }\end{array}$ & $\begin{array}{c}\text { Termina } \\
\text { tion } \\
\text { within } \\
30 \text { days }\end{array}$ & N/A & Yes & $\begin{array}{l}\text { Access } \\
\text { restrictio } \\
\mathrm{n} \text { then } \\
\text { terminat } \\
\text { ion }\end{array}$ \\
\hline $\begin{array}{c}\text { Local } \\
\text { /Foreign } \\
\text { Partnersh } \\
\text { ip }\end{array}$ & Yes & Yes. & Yes & Foreign & Yes & Foreign & Yes & Foreign & Yes & Foreign \\
\hline
\end{tabular}

\subsection{Security and Privacy Assessment}

According to the Table 2, the provider that ensures more security and privacy features towards client are Descasio, Just Host Name, followed by Cloud Exchange, Galaxy
Backbone and then Digital autonomy while the remaining providers do not lead in some of the features under this assessment

Table 2: Security and privacy features.

\begin{tabular}{|c|c|c|c|c|c|c|c|}
\hline $\begin{array}{l}\text { Organizati } \\
\text { on }\end{array}$ & $\begin{array}{l}\text { Data } \\
\text { Privacy } \\
\text { and } \\
\text { Loss }\end{array}$ & $\begin{array}{c}\text { Data } \\
\text { Integrity }\end{array}$ & $\begin{array}{c}\text { Proactive Threat } \\
\text { and } \\
\text { Vulnerability } \\
\text { Management }\end{array}$ & $\begin{array}{l}\text { Retention } \\
/ \\
\text { Dispositi } \\
\text { on }\end{array}$ & $\begin{array}{c}\text { Access } \\
\text { Control and } \\
\text { Privilege } \\
\text { Managemen } \\
\mathbf{t}\end{array}$ & $\begin{array}{l}\text { Preventi } \\
\text { ve } \\
\text { Measure } \\
\text { s }\end{array}$ & $\begin{array}{l}\text { Reactiv } \\
\text { e } \\
\text { Measur } \\
\text { es }\end{array}$ \\
\hline Layer3 & $\begin{array}{c}\text { Concern } \\
\text { ed }\end{array}$ & $\begin{array}{c}\text { Concern } \\
\text { ed }\end{array}$ & Not Concerned & $\begin{array}{c}\text { Concerne } \\
\mathrm{d}\end{array}$ & Concerned & $\begin{array}{c}\text { Moderat } \\
\mathrm{e}\end{array}$ & $\begin{array}{c}\text { Modera } \\
\text { te }\end{array}$ \\
\hline Mainone & $\begin{array}{c}\text { Not very } \\
\text { concerne } \\
\mathrm{d}\end{array}$ & $\begin{array}{c}\text { Not very } \\
\text { concerne } \\
d\end{array}$ & Not concerned & $\begin{array}{c}\text { Not } \\
\text { concerned }\end{array}$ & $\begin{array}{l}\text { Not very } \\
\text { concerned }\end{array}$ & $\begin{array}{l}\text { Inadequa } \\
\text { te }\end{array}$ & Poor \\
\hline CWG Plc & $\begin{array}{c}\text { Very } \\
\text { much } \\
\text { concerne } \\
\text { d }\end{array}$ & $\begin{array}{c}\text { Very } \\
\text { much } \\
\text { concerne } \\
\text { d }\end{array}$ & $\begin{array}{l}\text { Very much } \\
\text { concerned }\end{array}$ & $\begin{array}{c}\text { Very } \\
\text { much } \\
\text { concerned }\end{array}$ & $\begin{array}{l}\text { Very much } \\
\text { concerned }\end{array}$ & Good & Poor \\
\hline Descasio & $\begin{array}{c}\text { Very } \\
\text { much } \\
\text { concerne } \\
\mathrm{d}\end{array}$ & $\begin{array}{c}\text { Very } \\
\text { much } \\
\text { concerne } \\
\mathrm{d}\end{array}$ & $\begin{array}{l}\text { Very much } \\
\text { concerned }\end{array}$ & $\begin{array}{c}\text { Very } \\
\text { much } \\
\text { concerned }\end{array}$ & $\begin{array}{l}\text { Very much } \\
\text { concerned }\end{array}$ & Good & good \\
\hline $\begin{array}{c}\text { Whogohos } \\
\text { t }\end{array}$ & $\begin{array}{c}\text { Very } \\
\text { much } \\
\text { concerne } \\
\text { d }\end{array}$ & $\begin{array}{c}\text { Very } \\
\text { much } \\
\text { concerne } \\
\text { d }\end{array}$ & Very concerned & $\begin{array}{c}\text { Very } \\
\text { concerned }\end{array}$ & $\begin{array}{l}\text { Very much } \\
\text { concerned }\end{array}$ & Good & $\begin{array}{c}\text { moderat } \\
\mathrm{e}\end{array}$ \\
\hline $\begin{array}{c}\text { Just Host } \\
\text { Name }\end{array}$ & $\begin{array}{c}\text { Very } \\
\text { much } \\
\text { concerne } \\
\text { d }\end{array}$ & $\begin{array}{c}\text { Very } \\
\text { much } \\
\text { concerne } \\
\text { d }\end{array}$ & $\begin{array}{l}\text { Very much } \\
\text { concerned }\end{array}$ & $\begin{array}{c}\text { Very } \\
\text { much } \\
\text { concerned }\end{array}$ & $\begin{array}{l}\text { Very much } \\
\text { concerned }\end{array}$ & Good & Good \\
\hline $\begin{array}{c}\text { Cloud } \\
\text { Exchange }\end{array}$ & $\begin{array}{c}\text { Very } \\
\text { much } \\
\text { concerne } \\
\text { d }\end{array}$ & $\begin{array}{c}\text { Very } \\
\text { much } \\
\text { concerne } \\
\text { d }\end{array}$ & $\begin{array}{l}\text { Very much } \\
\text { concerned }\end{array}$ & $\begin{array}{c}\text { Very } \\
\text { much } \\
\text { concerned }\end{array}$ & $\begin{array}{l}\text { Very much } \\
\text { concerned }\end{array}$ & Basic & Good \\
\hline $\begin{array}{c}\text { Digital } \\
\text { Autonomy }\end{array}$ & $\begin{array}{c}\text { Very } \\
\text { much } \\
\text { concerne } \\
\text { d }\end{array}$ & $\begin{array}{c}\text { Very } \\
\text { much } \\
\text { concerne } \\
\text { d }\end{array}$ & $\begin{array}{l}\text { Very much } \\
\text { concerned }\end{array}$ & concerned & $\begin{array}{l}\text { Very much } \\
\text { concerned }\end{array}$ & Good & Good \\
\hline
\end{tabular}




\begin{tabular}{|c|c|c|c|c|c|c|c|}
\hline $\begin{array}{c}\text { Galaxy } \\
\text { Backbone }\end{array}$ & $\begin{array}{c}\text { Very } \\
\text { much } \\
\text { concerne } \\
\mathrm{d}\end{array}$ & $\begin{array}{c}\text { Very } \\
\text { much } \\
\text { concerne } \\
\mathrm{d}\end{array}$ & $\begin{array}{c}\text { Very much } \\
\text { concerned }\end{array}$ & $\begin{array}{c}\text { Very } \\
\text { much } \\
\text { concerned }\end{array}$ & $\begin{array}{c}\text { Very } \\
\text { concerned }\end{array}$ & Good & Basic \\
\hline Chert & $\begin{array}{c}\text { Very } \\
\text { much } \\
\text { concerne } \\
\mathrm{d}\end{array}$ & $\begin{array}{c}\text { Very } \\
\text { much } \\
\text { concerne } \\
\mathrm{d}\end{array}$ & $\begin{array}{c}\text { Very much } \\
\text { concerned }\end{array}$ & concerned & $\begin{array}{c}\text { Very } \\
\text { concerned }\end{array}$ & Good & Good \\
& & & & & \\
\hline
\end{tabular}

SECURITY \& PRIVACY ASSESSMENT

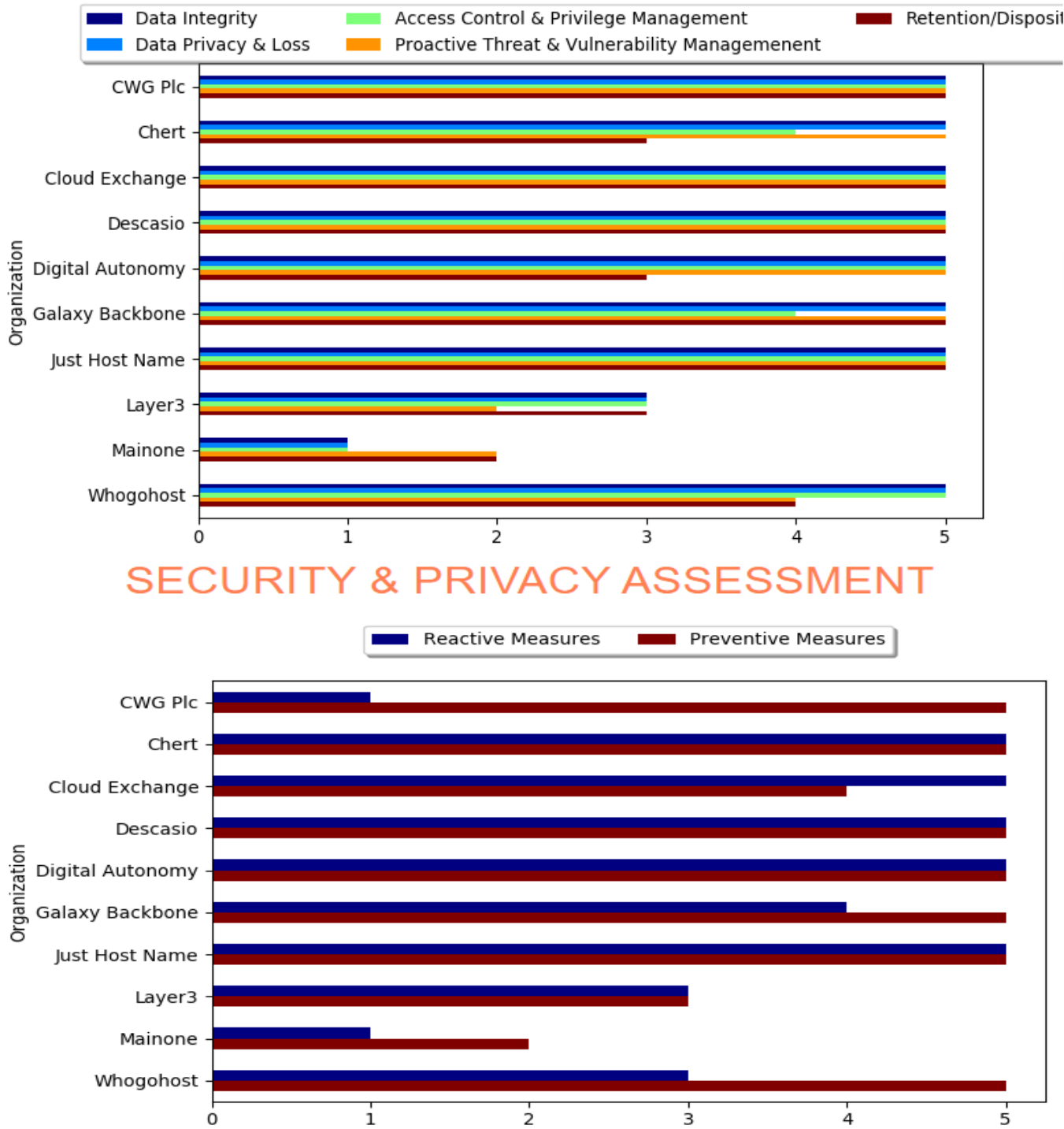

\subsection{Performance Assessment}

In Table 3, Layer3, CWG plc, Just Host Name, Cloud Exchange deploy more to their performance features, the next set of providers that follows are Whogohost, Digital
Autonomy, then Galaxy Backbone, Descasio while the remaining providers do not show much of these features in some areas.

Table 3: Performance assessment features.

\begin{tabular}{|c|c|c|c|c|}
\hline Organization & $\begin{array}{c}\text { Service } \\
\text { Response } \\
\text { Time }\end{array}$ & Accuracy & $\begin{array}{c}\text { Functional } \\
\text { ity }\end{array}$ & $\begin{array}{c}\text { Interoperabi } \\
\text { lity }\end{array}$ \\
\hline Layer3 & Good & Good & Good & Good \\
\hline Mainone & Poor & $\begin{array}{c}\text { Inadequat } \\
\mathrm{e}\end{array}$ & Inadequate & Inadequate \\
\hline
\end{tabular}




\begin{tabular}{|c|c|c|c|c|}
\hline CWG Plc & Good & Good & Good & Good \\
\hline Descasio & Basic & Basic & Basic & Basic \\
\hline Whogohost & Good & Good & Basic & Good \\
\hline $\begin{array}{c}\text { Just Host } \\
\text { Name }\end{array}$ & Good & Good & Good & Good \\
\hline $\begin{array}{c}\text { Cloud } \\
\text { Exchange }\end{array}$ & Good & Good & Good & Good \\
\hline $\begin{array}{c}\text { Digital } \\
\text { Autonomy } \\
\begin{array}{c}\text { Galaxy } \\
\text { Backbone }\end{array}\end{array}$ Good & Good & Good & Basic \\
\hline Chert & Good & Gasic & Good & Basic \\
\hline
\end{tabular}

\section{PERFORMANCE ASSESSMENT}

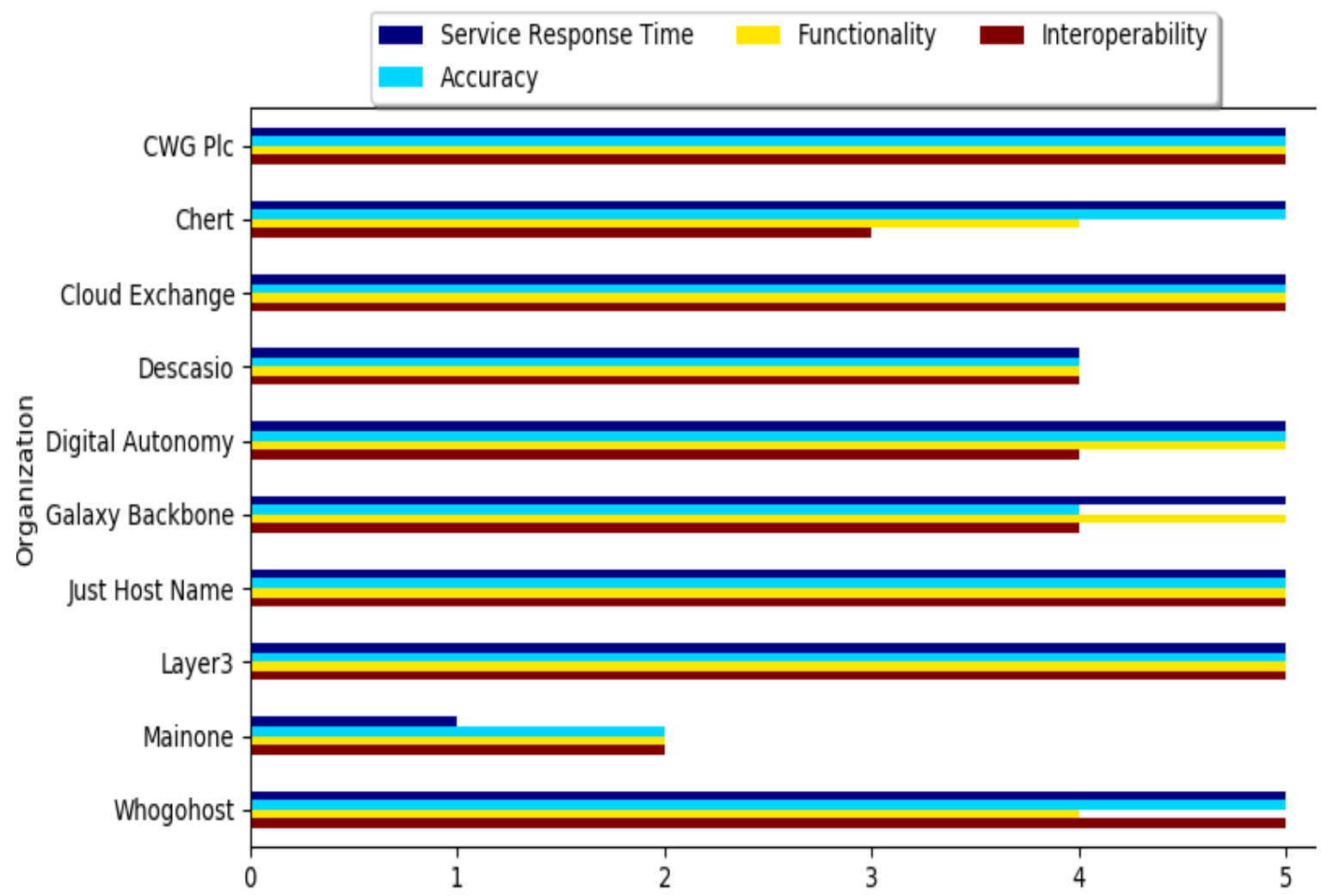

\subsection{Usability Assessment}

In this category, Providers such as CWG plc, Descasio, and Cloud Exchange excel in the usability features, followed by Just Host Name, Galaxy backbone, chert and

Table 4: Usability assessment features

\begin{tabular}{|c|c|c|c|}
\hline Organization & Customer Accessibility & Install Ability & Transparency \\
\hline Layer3 & Concerned & Concerned & Concerned \\
\hline Mainone & Not Concerned & Not Concerned & Not Concerned \\
\hline CWG Plc & Very Much Concerned & Very Much Concerned & Very Much Concerned \\
\hline Descasio & Very Much Concerned & Very Much Concerned & Very Much Concerned \\
\hline
\end{tabular}




\begin{tabular}{|c|c|c|c|}
\hline Whogohost & Very Concerned & Very Much Concerned & Concerned \\
\hline Just Host Name & Very Much Concerned & Very Concerned & Very Much Concerned \\
\hline Cloud Exchange & Very Much Concerned & Very Much Concerned & Very Much Concerned \\
\hline Digital Autonomy & Very Much Concerned & Very Concerned & Concerned \\
\hline Galaxy Backbone & Very Much Concerned & Very Concerned & Very Much Concerned \\
\hline Chert & Very Much Concerned & Very Much Concerned & Very Concerned \\
\hline
\end{tabular}

\subsection{Agility Assessment}

The provider that exercise more agility features based on the Table 5 are CWG Plc, Just Host Name, and Cloud

\section{Exchange}

followed by Descasio, Galaxy Backbone and then Whogohost, Digital Autonomy, and Chert.

Table 5: Agility assessment features.

\begin{tabular}{|c|c|c|c|c|c|}
\hline Organization & Adoptability & Elasticity & Extensibility & Scalability & Portability \\
\hline Layer3 & Concerned & Concerned & Concerned & Concerned & Concerned \\
\hline Mainone & Not Concerned & $\begin{array}{c}\text { Not Very } \\
\text { Concerned }\end{array}$ & Not Concerned & Not Concerned & Not Concerned \\
\hline CWG Plc & $\begin{array}{l}\text { Very Much } \\
\text { Concerned }\end{array}$ & $\begin{array}{l}\text { Very Much } \\
\text { Concerned }\end{array}$ & $\begin{array}{l}\text { Very Much } \\
\text { Concerned }\end{array}$ & $\begin{array}{l}\text { Very Much } \\
\text { Concerned }\end{array}$ & $\begin{array}{l}\text { Very Much } \\
\text { Concerned }\end{array}$ \\
\hline Descasio & Very Concerned & $\begin{array}{l}\text { Very Much } \\
\text { Concerned }\end{array}$ & $\begin{array}{l}\text { Very Much } \\
\text { Concerned }\end{array}$ & $\begin{array}{l}\text { Very Much } \\
\text { Concerned }\end{array}$ & Very Concerned \\
\hline Whogohost & Concerned & Very Concerned & Very Concerned & $\begin{array}{l}\text { Very Much } \\
\text { Concerned }\end{array}$ & $\begin{array}{l}\text { Very Much } \\
\text { Concerned }\end{array}$ \\
\hline $\begin{array}{l}\text { Just Host } \\
\text { Name }\end{array}$ & $\begin{array}{l}\text { Very Much } \\
\text { Concerned }\end{array}$ & $\begin{array}{l}\text { Very Much } \\
\text { Concerned }\end{array}$ & $\begin{array}{l}\text { Very Much } \\
\text { Concerned }\end{array}$ & $\begin{array}{l}\text { Very Much } \\
\text { Concerned }\end{array}$ & $\begin{array}{l}\text { Very Much } \\
\text { Concerned }\end{array}$ \\
\hline $\begin{array}{c}\text { Cloud } \\
\text { Exchange }\end{array}$ & $\begin{array}{l}\text { Very Much } \\
\text { Concerned }\end{array}$ & $\begin{array}{l}\text { Very Much } \\
\text { Concerned }\end{array}$ & $\begin{array}{l}\text { Very Much } \\
\text { Concerned }\end{array}$ & $\begin{array}{l}\text { Very Much } \\
\text { Concerned }\end{array}$ & $\begin{array}{l}\text { Very Much } \\
\text { Concerned }\end{array}$ \\
\hline $\begin{array}{c}\text { Digital } \\
\text { Autonomy }\end{array}$ & Very Concerned & Very Concerned & Very Concerned & Very Concerned & Not Concerned \\
\hline $\begin{array}{c}\text { Galaxy } \\
\text { Backbone }\end{array}$ & $\begin{array}{l}\text { Very Much } \\
\text { Concerned }\end{array}$ & Very Concerned & $\begin{array}{l}\text { Very Much } \\
\text { Concerned }\end{array}$ & Very Concerned & Very Concerned \\
\hline Chert & Very Concerned & $\begin{array}{l}\text { Very Much } \\
\text { Concerned }\end{array}$ & Concerned & $\begin{array}{l}\text { Very Much } \\
\text { Concerned }\end{array}$ & Very Concerned \\
\hline
\end{tabular}

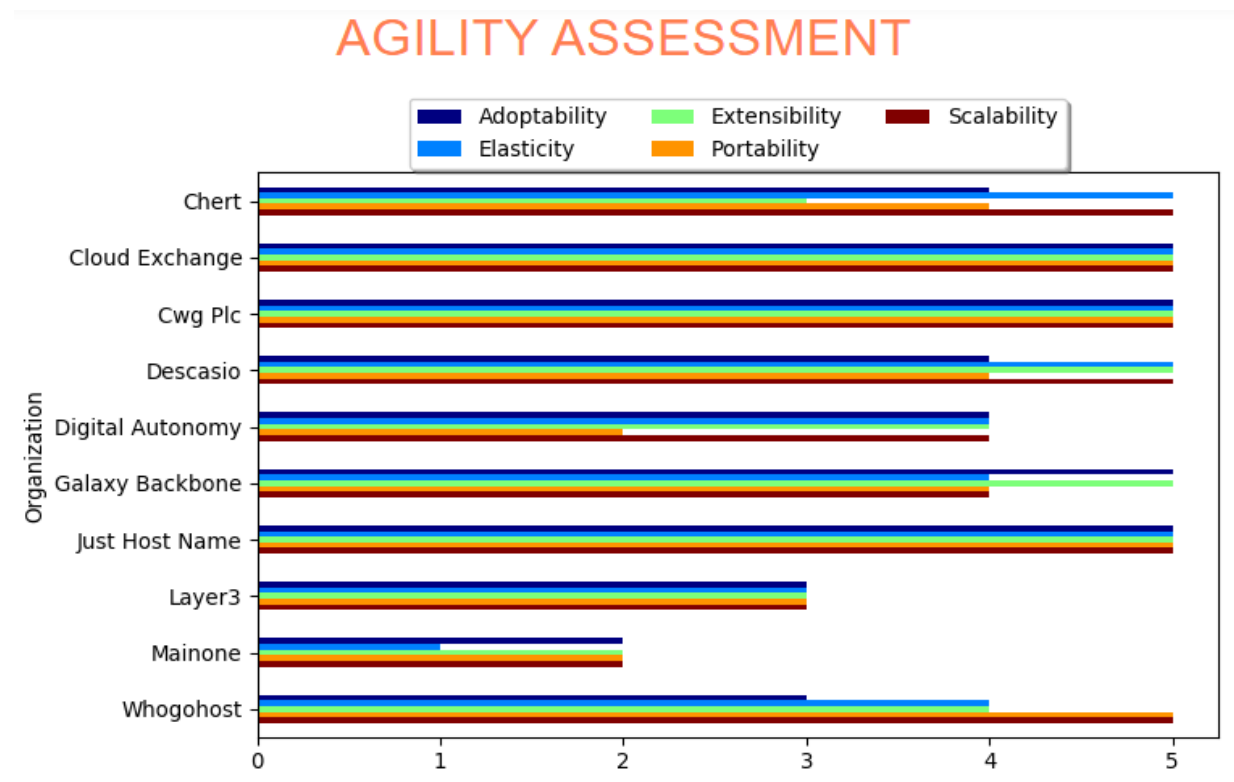




\subsection{Assurance Assessment}

Majority of the cloud computing providers establish good assurance features as shown in Table 6 except Chert,
Galaxy Backbone, Whogohost, And Mainone who do not scale up to the other providers based on some assessment qualities like Resiliency.

Table 6: Assurance assessment features.

\begin{tabular}{|c|c|c|c|c|}
\hline Organization & Reliability & Support & Maintainability & Resiliency \\
\hline Layer3 & Good & Good & Good & Good \\
\hline Mainone & Inadequate & Inadequate & Inadequate & Poor \\
\hline CWG PIc & Good & Good & Good & Good \\
\hline Descasio & Good & Good & Good & Good \\
\hline Whogohost & Basic & Good & Basic & Good \\
\hline Just Host Name & Good & Good & Good & Good \\
\hline Cloud Exchange & Good & Good & Good & Good \\
\hline Digital Autonomy & Good & Good & Good & Good \\
\hline Galaxy Backbone & Good & Good & Basic & Good \\
\hline Chert & Good & Good & Basic & Moderate \\
\hline
\end{tabular}

\section{ASSURANCE ASSESSMENT}

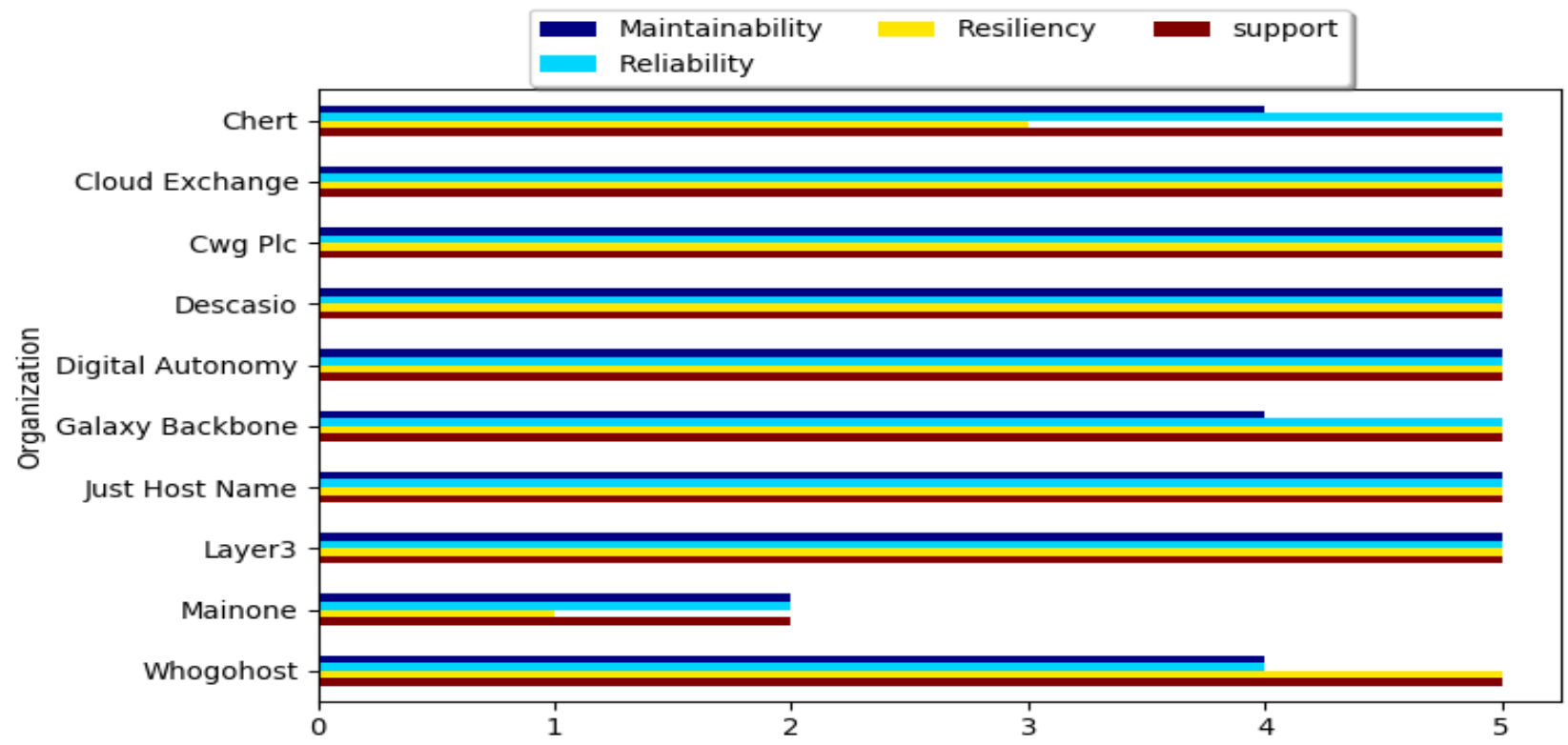

\subsection{Accountability Assessment}

Table 7 shows the providers that ensure more features in terms of accountability towards their clients. Examples are CWG Plc, Descasio, Just Host Name, Cloud Exchange, followed by Galaxy Backbone, Whogohost and then Digital autonomy while the remaining providers may have to deploy more into the areas of accountability.

Table 7: Accountability assessment features.

\begin{tabular}{|c|c|c|c|c|c|}
\hline Organization & Ownership & Governance & $\begin{array}{c}\text { Provider } \\
\text { Personnel } \\
\text { Requirement }\end{array}$ & Auditability & $\begin{array}{c}\text { Provider } \\
\text { Contact }\end{array}$ \\
\hline Layer3 & Concerned & Concerned & Concerned & Concerned & Concerned \\
\hline Mainone & $\begin{array}{c}\text { Not } \\
\text { Concerned }\end{array}$ & $\begin{array}{c}\text { Not Very } \\
\text { Concerned }\end{array}$ & Not Concerned & $\begin{array}{c}\text { Not } \\
\text { Concerned }\end{array}$ & $\begin{array}{c}\text { Not } \\
\text { Concerned }\end{array}$ \\
\hline Cwg Plc & $\begin{array}{c}\text { Very Much } \\
\text { Concerned }\end{array}$ & $\begin{array}{c}\text { Very Much } \\
\text { Concerned }\end{array}$ & $\begin{array}{c}\text { Very Much } \\
\text { Concerned }\end{array}$ & $\begin{array}{c}\text { Very Much } \\
\text { Concerned }\end{array}$ & $\begin{array}{c}\text { Very Much } \\
\text { Concerned }\end{array}$ \\
\hline
\end{tabular}




\begin{tabular}{|c|c|c|c|c|c|}
\hline Descasio & $\begin{array}{c}\text { Very Much } \\
\text { Concerned }\end{array}$ & $\begin{array}{c}\text { Very Much } \\
\text { Concerned }\end{array}$ & $\begin{array}{c}\text { Very Much } \\
\text { Concerned }\end{array}$ & $\begin{array}{c}\text { Very Much } \\
\text { Concerned }\end{array}$ & $\begin{array}{c}\text { Very Much } \\
\text { Concerned }\end{array}$ \\
\hline Whogohost & $\begin{array}{c}\text { Very Much } \\
\text { Concerned }\end{array}$ & Concerned & $\begin{array}{c}\text { Very Much } \\
\text { Concerned }\end{array}$ & Concerned & $\begin{array}{c}\text { Very } \\
\text { Concerned }\end{array}$ \\
\hline $\begin{array}{c}\text { Just Host } \\
\text { Name }\end{array}$ & $\begin{array}{c}\text { Very Much } \\
\text { Concerned }\end{array}$ & $\begin{array}{c}\text { Very Much } \\
\text { Concerned }\end{array}$ & $\begin{array}{c}\text { Very Much } \\
\text { Concerned }\end{array}$ & $\begin{array}{c}\text { Very Much } \\
\text { Concerned }\end{array}$ & $\begin{array}{c}\text { Very Much } \\
\text { Concerned }\end{array}$ \\
\hline $\begin{array}{c}\text { Cloud } \\
\text { Exchange }\end{array}$ & $\begin{array}{c}\text { Very Much } \\
\text { Concerned }\end{array}$ & $\begin{array}{c}\text { Very Much } \\
\text { Concerned }\end{array}$ & $\begin{array}{c}\text { Very Much } \\
\text { Concerned }\end{array}$ & $\begin{array}{c}\text { Very Much } \\
\text { Concerned }\end{array}$ & $\begin{array}{c}\text { Very Much } \\
\text { Concerned }\end{array}$ \\
\hline $\begin{array}{c}\text { Digital } \\
\text { Autonomy }\end{array}$ & $\begin{array}{c}\text { Very Much } \\
\text { Concerned }\end{array}$ & $\begin{array}{c}\text { Very } \\
\text { Concerned }\end{array}$ & $\begin{array}{c}\text { Very } \\
\text { Concerned } \\
\text { Concerned }\end{array}$ & Concerned \\
\hline $\begin{array}{c}\text { Galaxy } \\
\text { Backbone }\end{array}$ & $\begin{array}{c}\text { Very Much } \\
\text { Concerned }\end{array}$ & $\begin{array}{c}\text { Very Much } \\
\text { Concerned }\end{array}$ & $\begin{array}{c}\text { Very } \\
\text { Concerned }\end{array}$ & $\begin{array}{c}\text { Very Much } \\
\text { Concerned }\end{array}$ & $\begin{array}{c}\text { Very Much } \\
\text { Concerned }\end{array}$ \\
\hline Chert & Very Much & $\begin{array}{c}\text { Very } \\
\text { Concerned }\end{array}$ & Not Concerned & $\begin{array}{c}\text { Not } \\
\text { Concerned }\end{array}$ & $\begin{array}{c}\text { Not Very } \\
\text { Concerned }\end{array}$ \\
\hline
\end{tabular}

\section{ACCOUNTABILITY ASSESSMENT}

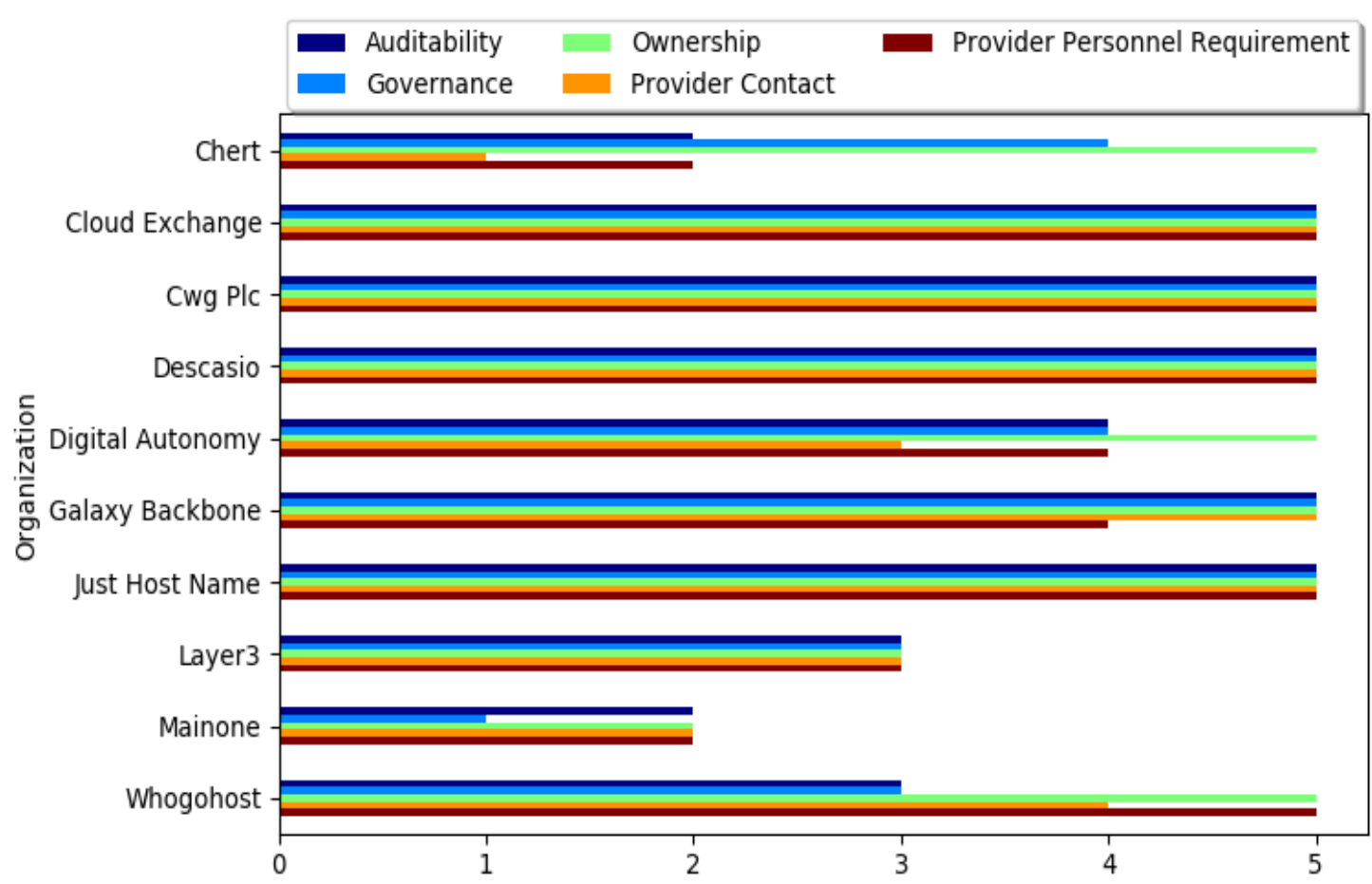

\section{CONCLUSION AND \\ RECOMMENDATION}

In conclusion, more indigenous CCPN were discovered and their features compared on various parameters whereby some providers outstand their peers in the various assessment segment they were examined. Generally, it is observed that larger percentage of the providers deploy more in the Security \& Privacy assessment study with Data Privacy \& Data Loss $(80.0 \%$ - very much concerned $)$, Data Integrity (80.0\% - very much concerned), Proactive Threat \& Vulnerability Management $(70 \%$ - very much concerned), Preventive Measure (70.0\% - Good), Access Control \& Privilege Management $(60.0 \%$ - very much concerned), Reactive Measure(50.0\% - Good), Retention/Disposition (50.0\% - very much concerned).

In terms of Performance assessment for the majority of respondents, the biggest concern is about Service Response Time $(80.0 \%$ - Good), furthermore next critical feature is Accuracy (70.0\% -Good), Functionality (60.0\% - Good), and Interoperability (50.0\% - Good).In terms of Usability assessment, the majority of respondents have indicated Customer Accessibility $(70.0 \%$ - very much concerned), followed by Install ability $(50.0 \%$ - very much concerned) and Transparency $(50.0 \%$ - very much concerned).

In terms of Agility assessment, most of the respondents indicated Scalability $(60.0 \%$ - very much concerned), Elasticity (50.0\% - very much concerned), Extensibility (50.0\% - very much concerned), Adoptability (40.0\% very much concerned), and Portability (40.0\% - very much concerned). In Assurance assessment, the most critical concern for the most of respondents is about Support (90.0\% - Good), Reliability (80.0\% - Good), 
Resiliency (80.0\% - Good), and Maintainability (60.0\% Good). While Accountability Assessment, the biggest concern for the majority of respondents is Ownership (80.0\% - very much concerned), followed by Governance (50.0\% - very much concerned), Provider Personnel Requirements $(50.0 \%$ - very much concerned), Auditability (50.0\% - very much concerned), and on the fifth place is Provider Contract $(50.0 \%$ - very much concerned).

It is recommended that further research can be centred on how providers should widen their scope by engaging in more awareness and educative scheme to the general public. Cloud computing providers and data centers should also increase their flexibility and automated processes wherein the customers need not worry about mundane concerns like upgrading software and accessibility around the globe.

\section{ACKNOWLEDGMENTS}

Our appreciation goes to the various CCPN who granted audience, made available to us useful information and ideas, and pieces of advice towards the success of this work.

\section{REFERENCES}

[1] Accenture, "Defense in the cloud," 2011. [Online]. Available:https://www.accenture.com/_acnmedia/pdf59/accenture-defense-cloud-pov.pdf. [Accessed 29 March 2019].

[2] Bhadauria, R., Sanyal, S. (2012). Survey on security issues in cloud computing and associated mitigation techniques. International Journal Computer Application, 47(18): 47-66. https://doi.org/10.5120/7292-0578

[3] Chert System Solutions Limited," 2011-2018. [Online]. Available: https://www.chert.ng/services. [Accessed 25 September 2019].

[4] Cisco Systems: 2009, Cisco cloud computing - Data Centre Strategy, Architecture, and Solutions., Point of view white paper for U.S. public sector, 1st edition.

[5] Cloud Exchange West Africa Website," [Online]. Available: https://www.cloudexchange.com.ng/cloudservices/. [Accessed 15 September 2019].
[6] CWG Plc, (2017). [Online]. Available: https://cwgplc.com/. [Accessed 5 August 2019].

[7] Descasio Limited, (2011). [Online]. Available: http://descasio.com/. [Accessed September 2019].

[8] Digital Autonomy Information Technology Website," 2019. [Online]. Available: https://www.daitng.com/about-us/. [Accessed 14 August 2019].

[9] Galaxy Backbone Limited," 2019. [Online]. Available: http://galaxybackbone.com.ng/iaas/. [Accessed 4 July 2019].

[10] Gance. T and Mell P, (2009): Effectively and Securely Using the Cloud Computing Paradigm, [Available online: https://ccsw.io/2009/slides/mell.pdf [Accessed 6 May 2020].

[11] Gartner: Gartner Highlights for Five Attributes of cloud Computing (2009) http://www.gartner.com/it/page.jsp?id=1035013. [Accessed 6 April 2019].

[12] Krutz, R.L., Vines, R.D. (2010). Cloud Security: A Comprehensive Guide to Secure Cloud Computing. Wiley Publishing Inc. Neww York.

[13] Landis, C and Blacharski, D. (2011): "Cloud computing made easy version 0.3," Available: http://www.rishabhcode.in/files/cloud_computing_ma de_easy.pdf

[14] Layer3: Layer3, 2005-2016. [Online]. Available: https://layer3.ng/about-layer3. [6 May 2020].

[15] Mainone, (2018). [Online]. Available: https://www.mdx-i.com/cloud/. [Accessed 4 July 2019].

[16] Ogunjobi M. (2015), "Barriers to Cloud Computing Adoption in Nigeria" The Guardian. Available at http://guardian.ng/technology/barriers-to-cloudcomputing-adoption-in-nigeria/ [Accessed 15 April 2019].

[17] T-Systems, "White paper cloud computing. Alternative sourcing strategy for business," 2013. 